\title{
A genetic replacement system for selection-based engineering of essential proteins
}

\author{
Sonja Billerbeck and Sven Panke*
}

\begin{abstract}
Background: Essential genes represent the core of biological functions required for viability. Molecular understanding of essentiality as well as design of synthetic cellular systems includes the engineering of essential proteins. An impediment to this effort is the lack of growth-based selection systems suitable for directed evolution approaches.

Results: We established a simple strategy for genetic replacement of an essential gene by a (library of) variant(s) during a transformation.

The system was validated using three different essential genes and plasmid combinations and it reproducibly shows transformation efficiencies on the order of $10^{7}$ transformants per microgram of DNA without any identifiable false positives. This allowed for reliable recovery of functional variants out of at least a $10^{5}$-fold excess of non-functional variants. This outperformed selection in conventional bleach-out strains by at least two orders of magnitude, where recombination between functional and non-functional variants interfered with reliable recovery even in recA negative strains.

Conclusions: We propose that this selection system is extremely suitable for evaluating large libraries of engineered essential proteins resulting in the reliable isolation of functional variants in a clean strain background which can readily be used for in vivo applications as well as expression and purification for use in in vitro studies.
\end{abstract}

\section{Background}

About eight percent of $E$. coli genes are essential for the cell [1]. Essential genes are of particular scientific interest as they encode proteins required for important biological functions, thereby building the minimal core of cellular viability which tends to be conserved across species. Knowledge about essential genes and their protein products is important for drug design [2,3], biotechnological applications [4], minimal genome approaches [5-8] and, in general, crucial for understanding and engineering the basic cellular functions required for life [9]. While the construction of the Keio-collection, a collection of single gene knock-outs in E. coli [1], enormously facilitated the systematic investigation of the physiology of $E$. coli as well as protein and strain-engineering approaches, it is still restricted to non-essential genes and their protein products. Engineering approaches involving essential genes

\footnotetext{
* Correspondence: sven.panke@bsse.ethz.ch

ETH Zürich, Department for Biosystems Science and Engineering (D-BSSE), Mattenstrasse 26, 4058 Basel, Switzerland
}

and proteins are complicated because knock-outs cause lethality. This means that phenotypes of engineered proteins cannot be easily evaluated in vivo as suitable clean strain backgrounds are not available.

In the last decade several approaches have been investigated to identify essential genes and to study their function in vivo by conditional elimination of the protein from the cell. This was achieved by triggering interference of the synthesis of the target protein on either the transcriptional or translational level [10-15]. However, these "bleach-out" methods rely on conditional protein elimination rather than elimination of the target gene itself and thus retain a wild-type copy of the essential gene in the cell. This sets limitations for the utility of these systems as ready-to-use selection systems for directed evolution experiments since recombination of library members with the chromosomal wild-type gene or mutations in the system regulating the expression of the wild-type protein can lead to the selection of false positive variants. This is particularly true when using a library for which only a small fraction of variants is expected to be functional. In this 
case recombination events are preferentially selected over functional library members, which results in every selection effort turning into a laborious screen for bona fide functional library members.

Besides evaluation of large libraries, another desire during protein engineering of essential genes is to replace the wild-type gene by a single engineered or heterologous variant for in vivo functional studies or for the construction of specialized strains which can be used to purify the mutant protein free of wild-type protein. Phage P1-mediated transduction of a chromosomal knock-out into a strain expressing a variant of the essential gene of interest from a plasmid is the current method of choice to achieve genetic replacement of an essential target gene by a variant (e.g [16]). The knockout was thereby created while complementing the chromosomal gene loss by a plasmid-encoded version of the essential gene.

Although P1-transduction is widely used, the protocol is time-consuming and restricted to a few variants at a time as efficiencies of successful transductions are low, often requiring empirical testing for the proper phage concentration followed by re-plating and PCR-screening for correct genotypes. Therefore, it was our aim to develop a general genetic set-up which turns working with essential genes and the engineering of their gene products into a straight-forward approach as facile as working with non-essential genes. Here, we present a simple transformation-based system. Establishment of the system begins with the chromosomal replacement of the essential gene of interest by a PCR-derived selection marker [17], in conjunction with a complementary vector-encoded version of the target. A central element of the method is that the complementation vector carries an I-SceI nuclease recognition site and can thus be rapidly and conditionally eliminated in the presence of an I-SceI nuclease-expressing helper plasmid. During elimination of the complementation vector, cells are made electrocompetent and transformed with a vectorencoded library or a specific variant of the essential gene. Thus, the actual step of gene exchange is reduced to a single transformation plus preparation of a suitable knock-out strain.

\section{Results}

\section{Overview of the replacement system}

The selection system can be flexibly assembled in various user-defined ways, but will be discussed in its simplest version first. It relies on two vectors: the knock-out and complementation vector pKOCOMP and the helper plasmid pI-SceI (Figure 1A and Table 1). Plasmid pKOCOMP is a derivative of pKD46 [17] and encodes the arabinose-inducible $\lambda$ red recombination system (genes $\beta, \gamma$ and exo) as well as the essential gene of interest. It also contains an 18 bp I-SceI cleavage site which allows for the conditional elimination of pKOCOMP in the presence of I-SceI. I-SceI is expressed from the helper plasmid pI-SceI under the control of the rhamnose-

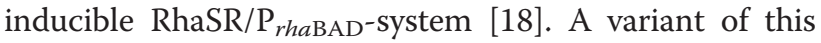
helper plasmid, $\mathrm{pP}_{\text {ara }} \mathrm{I}-\mathrm{Sce} \mathrm{I}$, carries an arabinoseinducible regulatory system $\mathrm{AraC} / \mathrm{P}_{\text {araBAD }}[19]$ instead of the rhamnose-based system. After testing various setups, these promoters were chosen as they exhibited high expression level in the presence of rhamnose or arabinose but could be efficiently switched off in the presence of glucose due to catabolite repression $[19,20]$. It should be noted that the specific set-up of the complementation vector is flexible as long as it carries the I-SceI site, which can easily be introduced by PCR, is compatible with pKD46, which is required for $\lambda$ red-mediated knock-out of the target gene and can be cured by growth at $42^{\circ} \mathrm{C}$. The system can then be completed by subsequent transformation with $\mathrm{pI}-\mathrm{Sce} \mathrm{I}$ or $\mathrm{pP}_{\text {ara }} \mathrm{I}-\mathrm{Sce}$.

\section{Validation and characterization of the system using the essential gene product adenylate kinase (Adk)}

The first target gene chosen for validation and characterization of the system was $a d k$, encoding E. coli's Adk, an essential gene product required for the biosynthesis of purine ribonucleotides and for the regulation of intracellular nucleotide availability $[1,21,22]$. For complementation, $a d k$ under control of $\mathrm{P}_{t a c}$ was inserted into

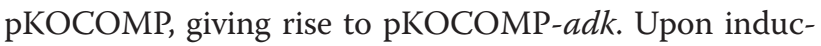
tion of pKOCOMP-adk with IPTG, the chromosomal copy of $a d k$ was replaced by a PCR-generated kanamycin resistance cassette [17]. The genotype of the resulting strain E. coli adk::kan [pKOCOMP-adk] was confirmed by PCR using primers binding to chromosomal regions up- and downstream of the adk-locus (Additional file 1: Table S1 and Additional file 2 Figure S1). To complete the selection system, strain adk::kan [pKOCOMP-adk] was transformed with helper plasmid pI-SceI. The resulting strain $a d k:: k a n$ [pKOCOMP-adk; pI-SceI] was grown in the presence of glucose during maintenance to repress I-SceI nuclease production. However, when cells were transferred to glucose-free LB medium, I-Scel production was apparently efficiently induced by the addition of rhamnose, as no colony-forming units could be recovered after an induction-8period of 180 minutes (Figure 2A). Importantly, even without a copy of the $a d k$ gene, cells remained viable for about two generations, presumably until all mRNA and protein was depleted. This was expected as linear DNA is rapidly degraded by cellular exonucleases and has a half-life in the range of minutes [23] whereas most proteins are relatively stable with half-lives in the range of hours [24].

During this intermediate period cells were made electrocompetent by washing with $\mathrm{H}_{2} \mathrm{O}$ and glycerol and 


\section{A}

Plasmids for knock-out and complementation
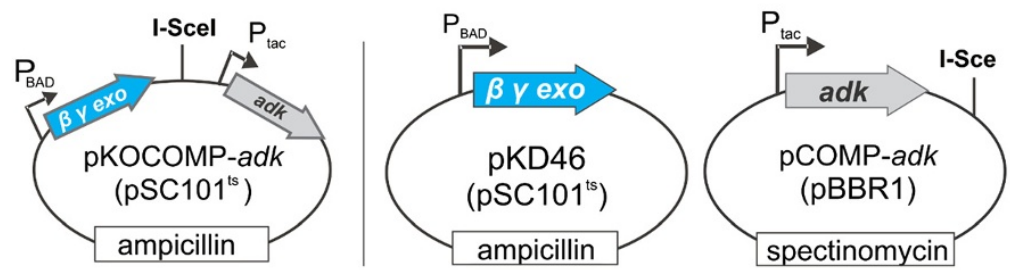

Helper plasmids:

expressing I-Scel-nuclease for conditional elimination of pKOCOMP or pCOMP

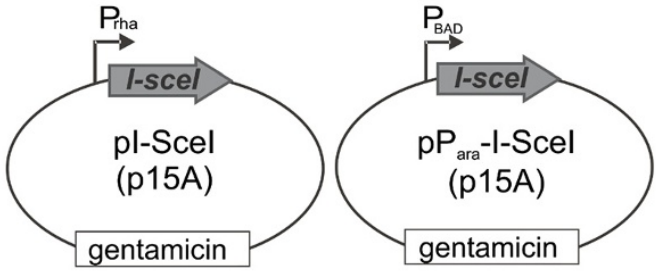

B

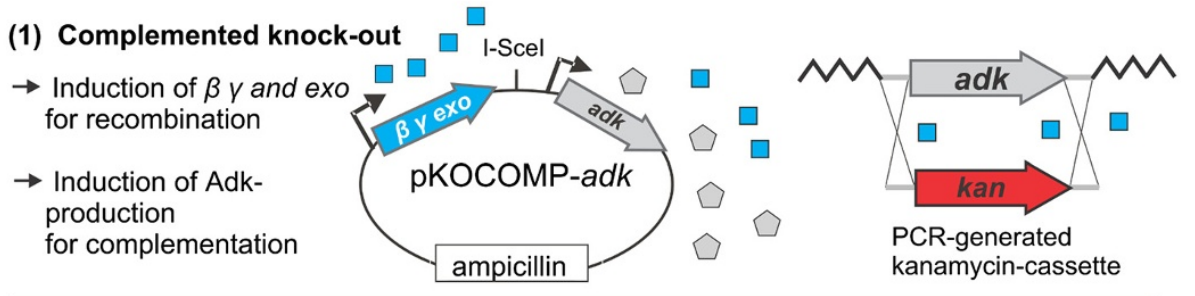

(2) Conditional elimination...

$\rightarrow$ Induction of Adk-production for complementation

$\rightarrow$ Induction of I-Scel-production to eliminate pKOCOMP-adk
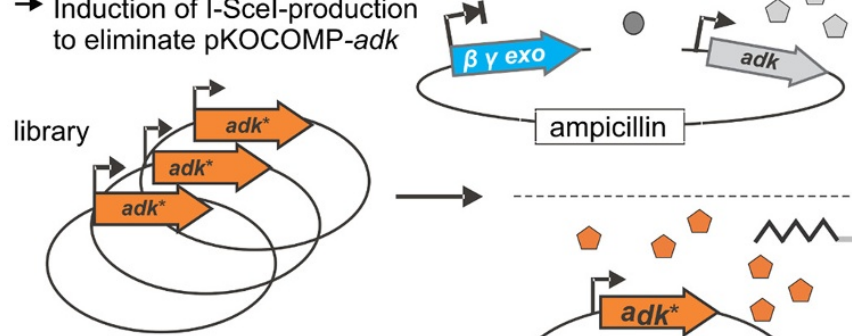

$M \stackrel{M a n}{\longrightarrow} M$
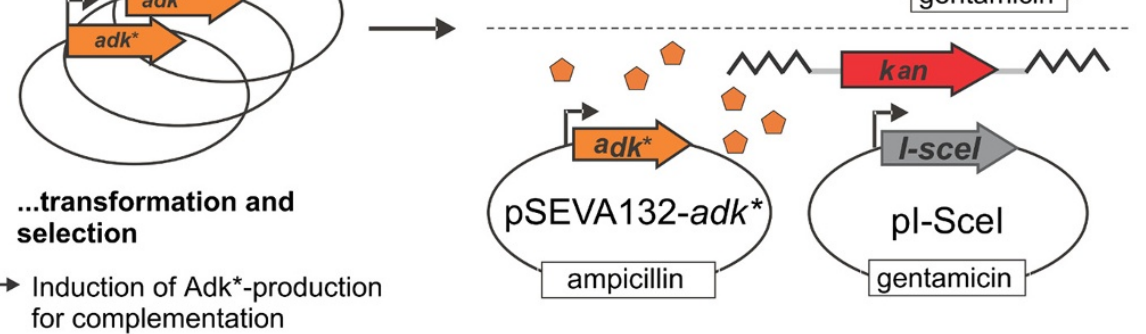

Figure 1 Overview on the transformation-based replacement of essential genes. A: Plasmids required for establishment of the selection system B: General procedure: The essential target gene adk is replaced by an antibiotic resistance cassette while pKOCOMP-adk complements for the chromosomal loss. Plasmid pKOCOMP can be conditionally eliminated by co-expression of I-Scel nuclease from helper plasmid pl-Scel. During elimination, cells are made electrocompetent and are transformed with a variant or library under investigation to evaluate functionality or to select for functional library members which can complement for the loss of pKOCOMP. 
Table 1 Bacterial strains and plasmids used in this study

\begin{tabular}{|c|c|c|}
\hline Bacterial strain or plasmid & Characteristics & Source \\
\hline \multicolumn{3}{|l|}{ Strains } \\
\hline W3110 & $F^{-} \lambda^{-} r p h-1 \mathrm{INV}(r r n D, r r n E)$ & $\begin{array}{l}\text { [33], internal } \\
\text { strain collection }\end{array}$ \\
\hline $\mathrm{DH} 10 \mathrm{~B}$ & 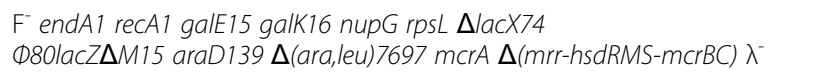 & $\begin{array}{l}\text { [34], internal } \\
\text { strain collection }\end{array}$ \\
\hline W3110 adk::kan & $\begin{array}{l}\text { Chromosomal adk replaced by a } \mathrm{Km}^{\mathrm{R}} \text { cassette. } \\
\text { The strain is only viable if the adk deletion is complemented. }\end{array}$ & This work \\
\hline W3110 groE::kan & $\begin{array}{l}\text { Chromosomal groS and groL replaced by a } \mathrm{Km}^{\mathrm{R}} \text { cassette. } \\
\text { The strain is only viable if the groE deletion is complemented. }\end{array}$ & This work \\
\hline $\begin{array}{l}\text { W3110 } \\
\text { secB-gpsA:.kan }\end{array}$ & $\begin{array}{l}\text { Chromosomal } \sec B \text { and gps } A \text { replaced by a } \mathrm{Km}^{R} \text { cassette. } \\
\text { The strain is only viable if the gps A deletion is complemented. }\end{array}$ & This work \\
\hline SB $\Delta$ recA & 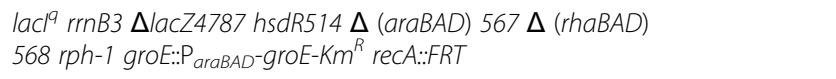 & This work \\
\hline \multicolumn{3}{|l|}{ Plasmids } \\
\hline pKD46 & $\begin{array}{l}\text { ori pSC } 101^{t s}, A p^{R} \text {. Encodes } \lambda \text { red recombination genes } \\
\gamma, \beta \text { and exo under control of the arabinose-responsive promoter } P_{\text {araBAD. }}\end{array}$ & [1] \\
\hline pKOCOMP-adk & $\begin{array}{l}\text { pKOCOMP-derived vector with adk under control of an } \\
\text { IPTG-responsive tac promoter }\end{array}$ & This work \\
\hline pSEVA432 & ori pBBR1, Spec ${ }^{R}$, multiple cloning site & $\begin{array}{l}\text { provided by } \\
\text { Victor de Lorenzo }\end{array}$ \\
\hline pCOMP-adk & $\begin{array}{l}\text { pSEVA432 encoding for adk under control of an IPTG-responsive } \\
\text { tac promoter, contains an I-Scel cleavage site }\end{array}$ & This work \\
\hline PCOMP-ESL & $\begin{array}{l}\text { pSEVA432 encoding for groS and grol control of an } \\
\text { IPTG-responsive tac promoter, contains an I-Scel cleavage site }\end{array}$ & This work \\
\hline pCOMP-secB-gpsA & $\begin{array}{l}\text { pSEVA432 encoding for the natural secB-gpsA transcriptional unit, } \\
\text { contains a I-Scel cleavage site }\end{array}$ & This work \\
\hline pSEVA132 & ori pBBR1, Ap ${ }^{R}$, multiple cloning site & $\begin{array}{l}\text { provided by } \\
\text { Victor de Lorenzo }\end{array}$ \\
\hline pSEVA132-adk & pSEVA132 encoding for adk under control of its natural promoter & This work \\
\hline pSEVA132-adk ${ }^{\text {Stop }}$ & pSEVA132-adk with an internal stop codon & This work \\
\hline pSEVA132-adk ${ }^{\text {watermark }}$ & pSEVA132-adk with a peptide insertion behind position P140 & This work \\
\hline pSEVA132-groE & $\begin{array}{l}\text { PSEVA } 132 \text { coding for groS and groL under control of their } \\
\text { natural promoter }\end{array}$ & This work \\
\hline pSEVA132-groE Etop $^{2}$ & pSEVA132-groE with an internal stop codon & This work \\
\hline pSEVA132-groE ${ }^{\text {watermark }}$ & pSEVA132-groE with a peptide insertion behind site 1301 & This work \\
\hline pSEVA132-secBgpsA & $\begin{array}{l}\text { pSEVA132 encoding for } \sec B \text { and gpsA under control of their } \\
\text { natural promoter }\end{array}$ & This work \\
\hline pSEVA132- secBgps A ${ }^{\text {Stop }}$ & pSEVA132- secBgpsA with an internal stop codon & This work \\
\hline pSEVA671 & ori p15A, Gm ${ }^{R}$, multiple cloning site & $\begin{array}{l}\text { provided by } \\
\text { Victor de Lorenzo }\end{array}$ \\
\hline pl-Scel & $\begin{array}{l}\text { pSEVA671, with I-Scel nuclease under control of the rhamnose } \\
\text { inducible promoter } P_{\text {Rha }} \text { and the response regulators } \\
\text { RhaS and RhaR, derived from the rhammnose metabolizing } \\
\text { transcriptional unit of E. coli. }\end{array}$ & This work \\
\hline pParal-Scel & $\begin{array}{l}\text { pl-Scel with } P_{\text {rhaBAD }} \text { and regulators RhaS and RhaR exchanged } \\
\text { by the arabinose promoter } P_{\text {araBAD }} \text { and the regulator araC }\end{array}$ & This work \\
\hline
\end{tabular}

transformed with various test plasmids (Table 1) in order to determine transformation efficiencies and the frequency of false positive variants. Vector pSEVA132-adk encoding the wild-type adenylate kinase under control of its natural promoter was used for determination of transformation efficiencies. Transformation efficiencies of $10^{7} \mu^{-1}$ DNA were routinely achieved. The system was intensively characterized regarding the frequency of false positive variants which could arise due to recombination of the library plasmid with residual linearized 

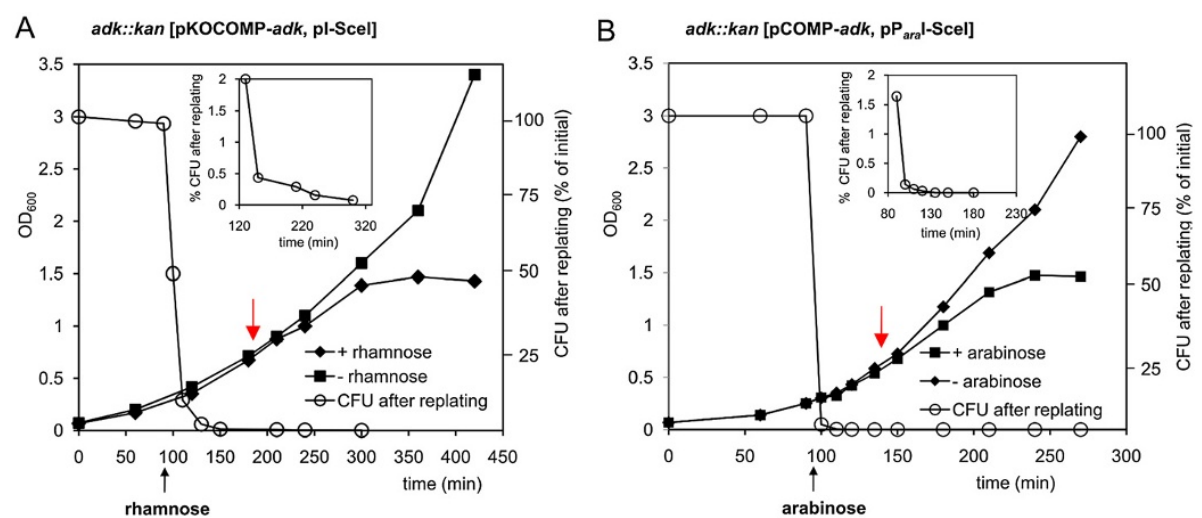

Figure 2 Characterization of the pKOCOMP-adk/pl-Scel and the pCOMP-adk/pP aral-Scel systems. A: Elimination of pKOCOMP-adk from adk::kan due to rhamnose-induced expression of I-Scel nuclease from helper plasmid pl-Scel. Red arrow: Time point for harvest and competent cell preparation. The inlet gives a better resolution of the elimination dynamics. The chromosomal adk-replacement was established using pKOCOMP-adk. B: Elimination of pCOMP-adk from adk:kan due to arabinose-induced expression of I-Scel nuclease from helper plasmid pP aral-Scel. The chromosomal adk-replacement was established using pKD46 and pCOMP-adk.

pKOCOMP-adk or due to an uninduced subpopulation of cells that maintained pKOCOMP (Table 2). Recombination-based false positives were tested by transformation with pSEVA132-adk ${ }^{\text {stop }}$, harboring a stop codon in the $a d k$ gene that could be repaired by recombination allowing growth of the corresponding carrier cell. False positives due to incomplete induction were examined by transformation with the empty vector pSEVA132. In both cases no false positive colonies were detected when I-SceI expression was induced and pKOCOMP was eliminated before competent cell preparation (Table 2), corresponding to a frequency of less than $2 \times 10^{-4}$ false positives per transformed cell. However, there was a detectable frequency of recombination events when pKOCOMP was eliminated only after transformation with the test plasmids or when we tried to eliminate pKOCOMP solely by growth at the nonpermissive temperature. In the former case, I-SceI nuclease expression was induced only after transformation by plating on arabinose-supplemented agar plates, such that both plasmids would be simultaneously present in the cells for a short period of time. Here we found illegitimate events with a frequency of about $3 \times 10^{-3}$ per transformed cell (Table 2). This demonstrates the importance of careful management of the plasmid elimination step.

\section{Flexibility of the replacement system}

To verify that the selection system can be set up with alternative combinations, we constructed pCOMP-adk. This vector is based on the pBBR1 ori with an expected copy number of 10-20 per cell [25], carrying a $\mathrm{P}_{t a c}$ promoter-controlled $a d k$ gene, the lac-repressor LacI, and an I-SceI cleavage site. While the previous complementation vector pKOCOMP- $a d k$ used a temperaturesensitive pSC101 ori with about 2-3 copies per cell when grown at $37^{\circ} \mathrm{C}$ [26], the increased copy number of pCOMP-adk allowed for examination of whether plasmid elimination was sufficiently efficient at higher intracellular plasmid concentration. We used pCOMP-adk in combination with pKD46 to replace $a d k$ by a kanamycin cassette. After curing cells of pKD46 by growth at $43^{\circ} \mathrm{C}$, the resulting strain $a d k:: k a n$ [pCOMP-adk] was transformed with $\mathrm{pP}_{\text {ara }} \mathrm{I}-\mathrm{Sce} \mathrm{I}$ and used for gene replacement as described before using arabinose to induce I-SceI production. Despite the higher copy number of pCOMP, the

Table 2 Transformation efficiencies and frequency of false positive variants for the Adk-specific selection systems

\begin{tabular}{|c|c|c|c|c|}
\hline \multirow[t]{2}{*}{ Strain } & \multirow[t]{2}{*}{$\begin{array}{l}\text { I-Scel induction during } \\
\text { competent cell preparation }\end{array}$} & \multicolumn{3}{|c|}{$\begin{array}{l}\text { Number of transformants } \\
\text { (frequency CFU per transformant) }\end{array}$} \\
\hline & & pSEVA132-adk & pSEVA132-adk ${ }^{\text {stop }}$ & pSEVA132 \\
\hline \multirow[t]{2}{*}{ W3110 adk::kan [рКОСОМP-adk, pl-Scel] } & No & $20200 \pm 2900$ & $66 \pm 21\left(\sim 3 \times 10^{-3}\right)$ & $28 \pm 13\left(\sim 1 \times 10^{-3}\right)$ \\
\hline & Yes & $21000 \pm 6000$ & $0\left(<2 \times 10^{-4}\right)$ & $0\left(<2 \times 10^{-4}\right)$ \\
\hline \multirow[t]{2}{*}{ W3110 adk::kan [pCOMP-adk, pP aral-Scel] } & No & $32100 \pm 3700$ & $61 \pm 17\left(2 \times 10^{-3}\right)$ & $35 \pm 11\left(1 \times 10^{-3}\right)$ \\
\hline & Yes & $28000 \pm 2200$ & $0\left(<2 \times 10^{-4}\right)$ & $0\left(<2 \times 10^{-4}\right)$ \\
\hline
\end{tabular}


plasmid was again rapidly eliminated from the cells in the presence of arabinose (Figure 2B). Probably due to the faster on-set and the possibly higher expression levels of the arabinose responsive promoter $\mathrm{P}_{\text {araBAD }}$ $[19,20]$, elimination-dynamics of the $\mathrm{pCOMP} / \mathrm{pP}$ ara $\mathrm{I}-$ SceI system were faster than those of the $\mathrm{pKOCOMP/pI-SceI}$ system. Although the elimination data of the two systems are difficult to compare due to differences in copy number and promoters, these results indicate that the described approach can be set up in multiple ways, making it easy to adapt to plasmid strategies for specific purposes. Transformation efficiencies of the $\mathrm{pCOMP} / \mathrm{pP}$ ara $\mathrm{I}-$ SceI system $\left(\sim 10^{7}\right.$ colonies $\mu \mathrm{g}^{-1}$ DNA $)$ were comparable to the $\mathrm{pKOCOMP/pI-SceI} \mathrm{system}$ (Table 1) and we could not identify false positive transformants.

\section{Generality of the system}

To confirm that the utility of the system was not limited to $a d k$ but could be easily extended to other essential genes, we constructed in vivo selection systems for other essential gene products: the chaperonin GroEL and its co-chaperonin GroES (encoded by the groE operon containing the genes groL and groS), and glycerol-3phosphate dehydrogenase (GpsA encoded by gpsA). For establishment of the GroEL-specific system we introduced an I-SceI cleavage site into the vector pSEVA431groE by PCR giving rise to vector pCOMP-groE. Plasmid pSEVA431-groE encodes the groE-operon under control of the IPTG-inducible $\mathrm{P}_{t a c}$ promoter. It also harbors the lacI gene, a spectinomycin resistance cassette and replicates with a pBBR1 ori. Plasmid pCOMP-groE was used in combination with pKD46 to replace the chromosomal groE-operon by a kanamycin resistance cassette (Additional file 2 Figure S1). The resulting strain groE::kan [pCOMP-groE] was cured from pKD46 at $43^{\circ} \mathrm{C}$ and then transformed with the helper plasmid $\mathrm{pP}_{\text {ara }} \mathrm{I}-S c e \mathrm{I}$.

The GpsA-specific system was constructed by cloning the natural gps $A$ transcriptional unit (consisting of genes $\sec B$ and gps $A$ under control of their natural promoter) into vector pSEVA431 using primers encoding for an ISceI restriction site, resulting in vector pCOMP$\operatorname{secBgps} A$. Chromosomally encoded $\sec B$ and gps $A$ were then replaced by a kanamycin resistance cassette using pKD46 (Additional file 2 Figure S1). To complete the set-up, strain secBgpsA::kan [pCOMP-secBgpsA] was cured of pKD46 and transformed with helper plasmid $\mathrm{pP}_{\text {ara }} \mathrm{I}-$ SceI. Both systems were characterized regarding elimination dynamics of the complementing plasmids pCOMP-groE and pCOMP-secBgpsA after I-SceI induction, as well as regarding transformation efficiencies of electrocompetent cells prepared during pCOMPelimination and frequency of false positive variants. Both pCOMP-type plasmids were lost at a comparable rate to the pCOMP-plasmid carrying adk (Figure 3). After that, both systems routinely showed transformation efficiencies of $10^{6}-10^{7}$ colonies $\mu^{-1}$ DNA when transformed with the positive control vectors pSEVA132-groE or pSEVA132-secBgpsA. No false positive variants could be detected after transformation with the test plasmids pSEVA132-groE stop $^{\text {and }}$ pSEVA132-secBgpsA $A^{\text {stop }}$, constructed in analogy to pSEVA132-adk ${ }^{\text {stop }}$ before. Importantly, it seems to be a general feature that cells stay viable - as judged by the doubling time in comparison to a non-induced control culture that did not produce I-SceI - for one or more generations, depending on the target gene, after loss of the complementing plasmid. This is an important characteristic of the system as cells can be made competent for transformation with a library or variant of an essential gene during a period where the complementing plasmid has already been lost and can no longer contribute to recombination.

\section{Performance of the system during selection}

To test the system regarding its performance in reliably identifying functional variants from a large library without contamination by false positive variants we challenged the GroEL- and the Adk-specific selection system with mock libraries with various excesses of non-functional variants. As there is a conventional bleach-out system available for GroEL (the E. coli MGM100 strain [10]), we also compared the performance of the replacement system developed here to the bleach-out system. In strain MGM100, the groE promoter has been replaced by the $\operatorname{araBAD}$ promoter and the regulatory gene $a r a C$. The strain can therefore be maintained in the presence of arabinose but GroEL production from the chromosomal locus can be fully repressed in the presence of glucose. This way, functional variants can be selected from a library in the presence of glucose. However, as a copy of the wild-type gene is still present during selection, recombination can lead to selection of false positive variants, which can dominate even in stringent selection conditions. To improve the strain regarding the expected recombination frequency we deleted $\operatorname{rec} A$ leading to the strain SB $\Delta r e c A$ (Additional file 2: Figure S1).

The created mock libraries contained decreasing ratios of functional to non-functional gene variants from $1: 10^{2}$ to $1: 10^{5}$ (functional:non-functional) mimicking libraries where a decreasing number of variants is expected to be functional within a large pool of non-functional variants, as it is typical e.g. for libraries created by error-prone PCR with different error rates. As non-functional variants we used pSEVA132-gro $E^{\text {stop }}$ or pSEVA132-adk ${ }^{\text {stop }}$. As functional variants we used pSEVA132-groE $E^{\text {watermark }}$ or pSEVA132-adk ${ }^{\text {watermark }}$. These plasmids encode for a GroEL or Adk-variant with an in-frame insertion of a short peptide at previously identified permissive sites. Both variants are fully functional and can be identified 

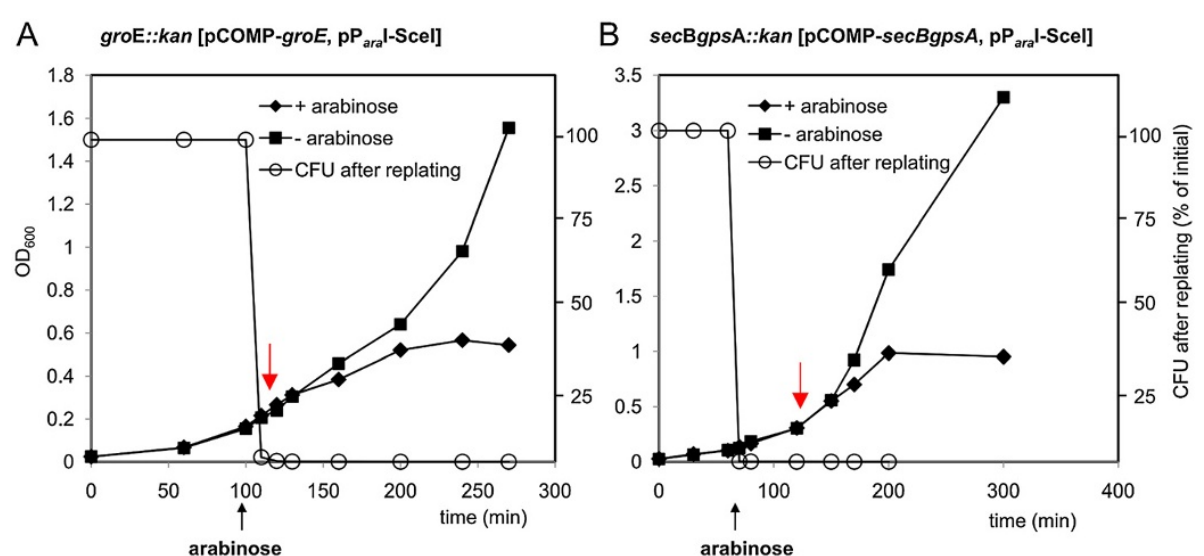

Figure 3 Characterization of the pCOMP-groE/pP aral-Scel and the pCOMP-secBgpsA/pParal-Scel system. Elimination of (A) pCOMP-groE from groE:.:kan and (B) pCOMP-secBgpsA from secBgpsA.:kan in the presence of arabinose and the helper plasmid pP aral-Scel. Elimination is induced by arabinose-induced expression of I-Scel nuclease from helper plasmid PP ara-Scel. Red arrow: Time point for harvest and competent cell preparation.

by PCR using watermark-specific primers. To compare the GroEL-specific selection system with the GroEL bleach-out system SB $\Delta r e c A$, electrocompetent W3110 groE::kan [pCOMP-groE, $\mathrm{pP}_{\text {ara }} \mathrm{I}-\mathrm{SceI}$ ] cells or electrocompetent $\mathrm{SB} \triangle \mathrm{rec} A$ cells were transformed with the different mock libraries in separate experiments. As positive and negative controls, cells were transformed with only functional or only non-functional variants. After plating and incubation overnight on LB-agar plates supplemented with arabinose, the corresponding antibiotics, and, in the case of W3110 groE::kan [pCOMPgroE, $\left.\mathrm{pP}_{\text {ara }} \mathrm{I}-\mathrm{Sce} \mathrm{I}\right]$, glucose, colonies were scored and a subset of the colonies was genotyped with watermarkspecific primers (Table 3).

Table 3 Recovery of functional GroEL-variants and Adk-variants from mock libraries with increasing excess of non-functional variants using the established selection system in comparison with a conventional bleach-out system

\begin{tabular}{|c|c|c|c|c|}
\hline Strain & Library (functional: non-functional) & $\begin{array}{c}\text { Number of colonies after } \\
\text { transformation }^{\mathrm{a}}\end{array}$ & $\begin{array}{c}\text { Number of genotyped } \\
\text { variants }\end{array}$ & $\begin{array}{l}\text { Number of detected } \\
\text { false positive variants }\end{array}$ \\
\hline \multirow{6}{*}{$\begin{array}{l}\text { W3110 groE::kan } \\
\text { [pCOMP-groE, pP aral-Scel] }\end{array}$} & Only functional & $\sim 27000$ & 20 & 0 \\
\hline & $1: 10^{2}$ & 231 & 20 & 0 \\
\hline & $1: 10^{3}$ & 33 & 20 & 0 \\
\hline & $1: 10^{4}$ & 4 & 4 & 0 \\
\hline & $1: 10^{5}$ & $1^{\mathrm{a}}$ & 1 & 0 \\
\hline & Only non-functional & 0 & 0 & 0 \\
\hline \multirow[t]{6}{*}{ SB $\Delta r e c A$} & Only functional & $\sim 25000$ & 20 & 0 \\
\hline & $1: 10^{2}$ & 253 & 20 & 0 \\
\hline & $1: 10^{3}$ & 25 & 20 & 1 \\
\hline & $1: 10^{4}$ & 7 & 7 & 5 \\
\hline & $1: 10^{5}$ & 6 & 6 & 5 \\
\hline & Only non-functional & 7 & 7 & 7 \\
\hline \multirow{6}{*}{$\begin{array}{l}\text { W3110 adk::kan } \\
\text { [pCOMP-adk, pP ara-Scel] }\end{array}$} & Only functional & $\sim 46000$ & 20 & 0 \\
\hline & $1: 10^{2}$ & 459 & 20 & 0 \\
\hline & $1: 10^{3}$ & 36 & 20 & 0 \\
\hline & $1: 10^{4}$ & 3 & 3 & 0 \\
\hline & $1: 10^{5}$ & 1 & 1 & 0 \\
\hline & Only non-functional & 0 & 0 & 0 \\
\hline
\end{tabular}

${ }^{\mathrm{a}}$ Typically, $10^{9}$ cells were used in the transformation, which would in some cases not have allowed to isolate a positive colony in the high-stringency case. In these cases, the number of transformed cells was increased to $3^{*} 10^{9}$ cells. 
In the GroEL-specific system, the numbers of colonies correlated well with the numbers expected from transformation efficiencies and the decreasing number of functional variants in each library. Even from the most stringent library (functional variants at a frequency of $10^{-5}$ ) only functional variants carrying the watermark were recovered after transformation of $\sim 81,000$ cells. No colony was detected after transformation of approximately the same number of cells with only nonfunctional variants. In contrast when using $\mathrm{SB} \Delta \mathrm{rec} A$ for the same experiment, false positive variants were detected after transformation with the negative control (transformation with only non-functional variants) with a frequency of $\sim 2 \times 10^{-4}$. In addition, we identified false positive variants after genotyping a subset of the colonies which had been selected from the different mock libraries (Table 3). Selection stringency positively correlated with the false positive rate, thus requiring laborious orthogonal assays to differentiate true from false positives. In a directed evolution experiment this would result in the requirement for intensive post-screening of selected variants for true functional library members. Plasmids of three of the false positive variants, which had been isolated after transformation with only nonfunctional variants, were further analyzed by sequencing. For two of them the stop codon had been reverted to the wild-type codon, probably due to recombination with the chromosomal groL copy. The third analyzed variant still had the stop codon within groL indicating that the ability to grow must have arisen from a mutation in the $\operatorname{araBAD}$ promoter preventing full repression. This phenomenon had been described before for strain MGM100 [16].

The same experiment was performed with the Adkspecific selection system using mock libraries with decreasing ratios of functional Adk-variants to non-functional Adk-variants. Also for this set-up we did not identify any false positive variant and after transformation with the most stringent library we could reliably identify a functional variant containing the watermark after transformation of $\sim 138,000$ cells (Table 3 ).

\section{Discussion}

In this study we present a facile and efficient set-up for a (within the tested boundaries) background-free selection system for functional, engineered, essential proteins. It is based on the conditional elimination of a complementary plasmid-based copy of an essential gene in a knockout strain in order to replace the essential gene by genes from a library (for example).

We show that I-SceI nuclease-based cleavage proves to be a suitable strategy for the fast and efficient elimination of a complementing plasmid from a knock-out strain which can occur while the strain is being made transformation-competent. Efficient plasmid elimination is an essential prerequisite for the high performance of the system during evaluation of large libraries under stringent conditions because elimination of the wild-type gene from the cells prior to introduction of variants prevents recombination-based background growth. The major shortcoming of conventionally used bleach-out systems is indeed due to recombination with a silenced wild-type gene during selection. Even in a $\operatorname{rec} A$ strain, RecA-independent recombination between homologous regions occurs with frequencies between $10^{-3}-10^{-4}$ [27]. As soon as the number of expected functional variants within a library drops below $10^{-3}$ selection is primarily for recombination events instead of functional library members. This can clearly be seen in our selection experiment using the GroEL bleach-out strain $\mathrm{SB} \Delta \mathrm{rec} A$ and mock libraries with decreasing ratio of functional variants. Even though our strain was deficient for $\operatorname{rec} A$, we frequently isolated false positive variants.

Besides being recombination-free, another advantage of the presented set-up over bleach-out systems is that selected variants are directly expressed in a clean strainbackground circumventing laborious post-transformation work such as P1-transductions. This allows for subsequent purification of engineered proteins for in vitro characterization and user-defined in vivo applications without running the risk of wild-type contamination.

We also show that even after elimination of the complementing vector from the knock-out strain, cells remain viable for one or more generations - depending on the gene - and electrocompetent cells prepared after elimination yield up to $10^{7}$ transformants $\mu \mathrm{g}^{-1}$ DNA.

As in our set-up the essential target genes are expressed from an inducible promoter during competent cell preparation, appropriate bleach-out times can be adjusted for individual gene products by tuning the inducer concentration.

The system was validated with three different essential $E$. coli proteins: Adk, glyceraldehyde-3-phosphate dehydrogenase and the chaperonin GroEL. Transformation efficiencies and the absence of detectable recombination events proved to be independent of the essential target gene.

\section{Conclusions}

In the current work we present a straightforward, transformation-based system which enables the genetic replacement of a wild-type essential gene of interest by a library or variant. It thereby directly results in the isolation of functional variants in a clean strain background with considerably reduced effort.

Furthermore, it substantially facilitates working with and engineering of essential genes and their protein products making it an experimentally easy, fast and scalable task. 
Finally, it should be possible to adapt the here introduced replacement strategy to other hosts - like e.g. yeast, Bacillus subtilis or Clustridium spec. - for which homologous recombination-based knock-out strategies are available [28-30].

\section{Materials and methods Chemical and enzymes}

Restriction enzymes and ligase were obtained from New England Biolabs (Ipswich, MA, USA) and used according to manufacturers' instructions. Chemicals were purchased in the highest purity available from Sigma-Aldrich, Fluka (Buchs, Switzerland) or Roth (Lauterbourg, France). Trypton and yeast extract were from BD Bioscience (Basel, Switzerland). Oligonucleotides and Sanger-sequencing service were purchased from Microsynth (Balgach, Switzerland).

\section{Strains and plasmids}

E. coli DH10B was used for general cloning procedures. E. coli W3110 was used as the chassis for all chromosomal deletions (see Table 1 for an overview on strains used in this study). $\mathrm{SB} \Delta \mathrm{rec} A$ is a derivative of strain MGM100 [10]. It was constructed from BW25113 $\operatorname{rec} A::$ FRT [1] by P1-phage transduction using a lysate from MGM100 and selecting for $K \mathrm{~m}^{R}$. The final clone was confirmed by PCR analysis of the groE and recA::FRT locus and by its inability to grow on glucose after GroEL-bleach-out. Plasmid pKOCOMP-adk is a derivative of pKD46 [17] and was constructed by first cloning the $a d k$ gene into the multiple cloning site of expression vector pACT3 [31] via restriction sites BamHI and HindIII using primers pKOCOMP-adk-fw and pKOCOMP$a d k$-rv (see Additional file 1 Table S1 for primer sequences). The resulting vector $\mathrm{pACT}-a d k$ was used as template to amplify the Ptac promoter-controlled adk gene with primers pACT-forward and pACT-reverse, encoding for a I-SceI recognition site, and cloned into pKD46 via its unique NcoI-site. For construction of pCOMP-adk the $\mathrm{P}_{t a c}$ promoter-controlled $a d k$ gene was amplified with primers pACT-SceI-Spe and pACT-Pac and the PCR product was cloned into the unique SpeI and PacI sites of pSEVA432 (ori pBBR1, Spec ${ }^{\mathrm{R}}$ resistance). Plasmid pSEVA132-adk is a derivative of pSEVA132 (ori pBBR1, Ap ${ }^{\mathrm{R}}$ ) and encodes $a d k$ controlled by its natural promoter and fused to a C-terminal $6 x$ His-tag. It was amplified from genomic $E$. coli DNA using primers $a d k$-forward and $a d k$-reverse and cloned into pSEVA132 via restriction sites $\mathrm{XmaI}$ and SacI. Plasmid pCOMP-groE was constructed by amplification of the $\mathrm{P}_{t a c}$-controlled groE-operon from pACT-ESL using primers pACT-SceI-Spe and pACT-Pac and cloned into the unique SpeI and PacI sites of pSEVA431. pACT-ESL is pACT3 derived and encodes the $\mathrm{P}_{t a c}$ controlled groE operon. groS was amplified from W3110 by PCR with primers groS-fw and groS-rv and groL was PCRamplified with primers groL-fw and groL-rv and then sequentially cloned into the KpnI and HindIII sites of pACT3. Plasmid pSEVA132-groE was constructed by amplification of the natural groE operon from $E$. coli genomic DNA using primers groE-forward and groEreverse and cloning them into the unique sites $X m a \mathrm{I}$ and $X b a I$. Plasmid pCOMP-secBgps $A$ was constructed by cloning the natural $\sec B$-gps $A$ transcriptional unit, amplified with primers secBgpsA-forward and gpsA_I-SceI-reverse, into pSEVA431 via restriction sites $X m a \mathrm{I}$ and $X b a \mathrm{I}$. Plasmids pSEVA132-adk ${ }^{\text {stop }}$, pSEVA132-adk ${ }^{\text {watermark }}$, pSEVA132-groE ${ }^{\text {stop }}$, pSEVA132-groE $E^{\text {watermark }}$ and pSEVA132-secBgps $\mathrm{A}^{\text {stop }}$ were constructed by amplification and re-ligation of pSEVA132-adk, pSEVA132-groE or pSEVA132-secBgpsA using primers adk-stop-fw/ $a d k$-stop-rv, $a d k$-watermark-fw/adk-watermark-rv, groEwatermark-fw/groE-watermark-rv, groE-stop-fw/groEstop-rv and secBgpsA-stop-fw/secBgpsA-stop-rv.

Helper plasmids $\mathrm{pI}-\mathrm{Sce} \mathrm{I}$ and $\mathrm{pP}_{\text {ara }} \mathrm{I}-\mathrm{Sce} \mathrm{I}$ are derivatives of pSEVA671 (ori p15A, Gm ${ }^{\mathrm{R}}$ ). The gene for I-SceI nuclease was amplified from plasmid pSTKST [32] using primers I-SceI-fw and I-SceI-rv and cloned into pSEVA671 via PacI and EcoRI restriction sites. The RhaR-RhaS $/ \mathrm{P}_{\text {rhaBAD }}$ regulatory system was amplified from E. coli genomic DNA using primers Rha-forward and Rha-reverse and cloned in front of I-SceI via restriction sites $N s i$ and SpeI. The AraC/ParaBAD regulatory system was amplified from $E$. coli genomic DNA using primers ParaBAD-fw and ParaBAD-rv and used to exchange RhaR-RhaS $/ \mathrm{P}_{r h a}$ using sites Nsi and SpeI.

\section{Preparation of competent cells and transformation}

E. coli adk::kan [pKOCOMP-adk, pI-SceI] or [pCOMP$\left.a d k, \mathrm{pP}_{\text {ara }} \mathrm{I}-\mathrm{Sce} \mathrm{I}\right]$ were grown overnight in LB liquid broth supplemented with $50 \mu \mathrm{g} \mathrm{mL} \mathrm{m}^{-1}$ kanamycin, $10 \mu \mathrm{g}$ $\mathrm{mL}^{-1}$ gentamicin, $100 \mu \mathrm{M}$ IPTG and $0.5 \%$ (wt/vol) glucose (to efficiently repress I-SceI production) at $30^{\circ} \mathrm{C}$ or $37^{\circ} \mathrm{C}$. Cells were pelleted, washed once with LB and diluted 1:100 in fresh LB broth, supplemented with the same antibiotics as before but without glucose. Cells were grown at $37^{\circ} \mathrm{C}$. At an $\mathrm{OD}_{600}$ of $0.2,10 \mathrm{mM}$ rhamnose or $0.2 \%$ arabinose (wt/vol) was added to induce ISceI nuclease production. When reaching an $\mathrm{OD}_{600}$ of 0.4-0.5, cells were chilled on ice for $30 \mathrm{~min}$, harvested and washed twice with chilled water and once with $10 \%$ glycerol as described [26]. For transformation with the test plasmids and the mock libraries, $50 \mu \mathrm{L}$ cells $\left(\mathrm{OD}_{600}\right.$ around 100) were mixed with $1.5 \mathrm{ng}$ DNA, exposed to an electrical pulse of $1.3 \mathrm{kV}$ and recovered in $1 \mathrm{~mL} \mathrm{LB}$ broth supplemented with $10 \mathrm{mM}$ rhamnose or $0.2 \%$ arabinose for $1 \mathrm{~h}$ at $37^{\circ} \mathrm{C}$. Selection was done overnight at $37^{\circ} \mathrm{C}$ on LB agar plates containing $50 \mu \mathrm{g} \mathrm{mL}$ 
kanamycin, $10 \mu \mathrm{g} \mathrm{mL}^{-1}$ gentamicin, $100 \mu \mathrm{g} \mathrm{mL}^{-1}$ ampicillin and $10 \mathrm{mM}$ rhamnose or $0.2 \%$ arabinose. The GroEL-specific selection system based on W3110 groE:: kan [pCOMP-groE, pP ara I-SceI] and the GpsA-specific selection system based on W3110 secBgpsA::kan [pCOMP-secBgpsA, $\mathrm{pP}_{\text {ara }} \mathrm{I}-\mathrm{SceI}$ ] were treated the same way. For preparation of competent $\mathrm{SB} \Delta r e c \mathrm{~A}$, cells were grown overnight in LB supplemented with $50 \mu \mathrm{g} \mathrm{mL}^{-1}$ kanamycin and $0.2 \%$ arabinose. Cells were washed twice with water and diluted 1:100 in fresh LB medium. When cells reached an $\mathrm{OD}_{600}$ of $0.10 .5 \%$ glucose was added to repress chromosomal GroEL production. Cellular GroEL was bleached for two generations before cells were harvested for competent cell preparation (at $\mathrm{OD}_{600}$ of 0.4).

\section{Determination of plasmid loss}

Loss of pKOCOMP-adk or pCOMP-adk, pCOMP-groE and pCOMP-secBgpsA was determined as follows: The corresponding knock-out strains adk::kan, groE::kan and secBgpsA::kan containing helper plasmid pI-SceI or $\mathrm{pP}_{\text {ara }} \mathrm{I}$-SceI were grown in LB liquid broth supplemented with $50 \mu \mathrm{g} \mathrm{mL}^{-1}$ kanamycin and $10 \mu \mathrm{g} \mathrm{mL}^{-1}$ gentamicin until exponential growth was reached. Next, I-SceI nuclease production was induced with $10 \mathrm{mM}$ rhamnose or $0.2 \%$ arabinose. After induction aliquots were taken after $0,10,20,40,60,120$ and $180 \mathrm{~min}$ and normalized to $\mathrm{OD}_{600}$. Serial dilutions were subsequently plated on LB agar supplemented with $0.5 \%$ glucose. The next day the number of colony forming units (CFU) was counted. The number of colonies resulting from aliquots which had been taken from a control culture grown in the absence of rhamnose or arabinose were set to $100 \%$ CFU.

\section{Chromosomal knock-out of essential genes}

Knock-outs were done by $\lambda$ red-based recombination with a PCR-encoded kanamycin resistance cassette as described earlier [17]. The kanamycin-cassettes were generated with pKD13 as a template and primers $a d k-$ $\mathrm{H} 1$ and $a d k-\mathrm{H} 2$, groE-H1 and groE-H2 or secBgpsA-H1 and secBgpsA-H2. Knock-outs were PCR-verified with primers P1-P6 as indicated in Additional file 2 Figure S1 and Additional file 1 Table S1.

\section{Additional files}

Additional files file 1: Table S1. Primers used in this study. Additional files file 2: Figure S1. PCR verification of knock out strains.

\section{Competing interests}

The authors declare that there are no competing interests.
Authors' contributions

SB performed the experiments, SB and SP designed the experimental approach and wrote the manuscript, SP supervised the research. All authors read and approved the final manuscript.

\section{Acknowledgment}

This work was funded by the EU FP6 projects Eurobiosyn and NANOMOT and the ESF project Nanocell. The authors are indebted to Tania Roberts for proofreading of the manuscript.

Received: 13 June 2012 Accepted: 8 August 2012

Published: 16 August 2012

\section{References}

1. Baba T, Ara T, Hasegawa M, Takai Y, Okumura Y, Baba M, Datsenko KA, Tomita M, Wanner BL, Mori H: Construction of Escherichia coli K-12 in-frame, single-gene knockout mutants: the Keio collection. Mol Syst Biol 2006, 2:2006-0008.

2. Rosamond J, Allsop A: Harnessing the power of the genome in the search for new antibiotics. Science 2000, 287:1973-1976.

3. Haselbeck R, Wall D, Jiang B, Ketela T, Zyskind J, Bussey H, Foulkes JG, Roemer T: Comprehensive essential gene identification as a platform for novel anti-infective drug discovery. Curr Pharm Des 2002, 8:1155-1172.

4. Johnson DBF, Xu JF, Shen ZX, Takimoto JK, Schultz MD, Schmitz RJ, Xiang Z, Ecker JR, Briggs SP, Wang L: RF1 knockout allows ribosomal incorporation of unnatural amino acids at multiple sites. Nat Chem Biol 2011, 7:779-786.

5. Gil R, Silva FJ, Pereto J, Moya A: Determination of the core of a minimal bacterial gene set. Microbiol Mol Biol Rev 2004, 68:518-537.

6. Posfai G, Plunkett G 3rd, Feher T, Frisch D, Keil GM, Umenhoffer K, Kolisnychenko V, Stahl B, Sharma SS, de Arruda M, et al: Emergent properties of reduced-genome Escherichia coli. Science 2006, 312:1044-1046.

7. Trinh CT, Unrean P, Srienc F: Minimal Escherichia coli cell for the most efficient production of ethanol from hexoses and pentoses. Appl Environ Microbiol 2008, 74:3634-3643.

8. Lee JH, Sung BH, Kim MS, Blattner FR, Yoon BH, Kim JH, Kim SC: Metabolic engineering of a reduced-genome strain of Escherichia coli for L-threonine production. Microb Cell Fact 2009, 8:2

9. Danchin A: Natural selection and immortality. Biogerontology 2009, 10:503-516.

10. McLennan N, Masters M: GroE is vital for cell-wall synthesis. Nature 1998 392:139-139.

11. Herring CD: Introduction of conditional lethal amber mutations in Escherichia coli. Methods Mol Biol 2008, 416:323-334.

12. Jin Y, Watt RM, Danchin A, Huang JD: Use of a riboswitch-controlled conditional hypomorphic mutation to uncover a role for the essential csrA gene in bacterial autoaggregation. J Biol Chem 2009, 284:28738-28745.

13. Ji Y, Zhang B, Van SF, Horn, Warren P, Woodnutt G, Burnham MK, Rosenberg M: Identification of critical staphylococcal genes using conditional phenotypes generated by antisense RNA. Science 2001, 293:2266-2269.

14. Tang YC, Chang HC, Chakraborty K, Hartl FU, Hayer-Hartl M: Essential role of the chaperonin folding compartment in vivo. EMBO J 2008, 27:1458-1468.

15. Herring CD, Blattner FR: Conditional lethal amber mutations in essential Escherichia coli genes. J Bacterio/ 2004, 186:2673-2681.

16. van der Vies SM, Lund PA: Determination of chaperonin activity in vivo. Methods Mol Biol 2000, 140:75-96.

17. Datsenko KA, Wanner BL: One-step inactivation of chromosomal genes in Escherichia coli K-12 using PCR products. Proc Natl Acad Sci U S A 2000 97:6640-6645

18. Tobin JF, Schleif RF: Transcription from the rha operon psr promoter. J Mol Biol 1990, 211:1-4.

19. Guzman LM, Belin D, Carson MJ, Beckwith J: Tight regulation, modulation, and high-level expression by vectors containing the arabinose PBAD promoter. J Bacteriol 1995, 177:4121-4130.

20. Egan SM, Schleif RF: A regulatory cascade in the induction of rhaBAD J Mol Biol 1993, 234:87-98.

21. Cousin D, Buttin G: Thermosensitive mutants of K12 Escherichia coli. 3. A lethal mutation of $E$. coli affecting the activity of the adenylate kinase. Ann Inst Pasteur (Paris) 1969, 117:612-630.

22. Glaser M, Nulty W, Vagelos PR: Role of adenylate kinase in the regulation of macromolecular biosynthesis in a putative mutant of Escherichia coli 
defective in membrane phospholipid biosynthesis. J Bacterio/ 1975, 123:128-136.

23. Kuzminov A, Schabtach E, Stahl FW: Chi sites in combination with RecA protein increase the survival of linear DNA in Escherichia coli by inactivating exoV activity of RecBCD nuclease. EMBO J 1994, 13:2764-2776.

24. Tobias JW, Shrader TE, Rocap G, Varshavsky A: The N-end rule in bacteria. Science 1991, 254:1374-1377.

25. Antoine $\mathrm{R}$, Locht $\mathrm{C}$ : Isolation and molecular characterization of a novel broad-host-range plasmid from Bordetella bronchiseptica with sequence similarities to plasmids from gram-positive organisms. Mol Microbiol 1992, 6:1785-1799.

26. Sambrook J, Russell DW: Molecular cloning: a laboratory manual. 3rd edition. Cold Spring Harbor, N.Y.: Cold Spring Harbor Laboratory Press; 2001.

27. Bi X, Liu LF: RecA-independent and RecA-dependent intramolecular plasmid recombination - Differential homology requirement and distance effect. J Mol Biol 1994, 235:414-423.

28. Wang Y, Weng J, Waseem $R$, Yin X, Zhang R, Shen Q: Bacillus subtilis genome editing using ssDNA with short homology regions. Nucleic Acids Res 2012, 40:e91.

29. Kuehne SA, Minton NP: ClosTron-mediated engineering of Clostridium. Bioengineered 2012, 3:245-252.

30. Wach A, Brachat A, Pohlmann R, Philippsen P: New heterologous modules for classical or PCR-based gene disruptions in Saccharomyces cerevisiae. Yeast 1994, 10:1793-1808.

31. Dykxhoorn DM, St Pierre R, Linn T: A set of compatible tac promoter expression vectors. Gene 1996, 177:133-136.

32. Kolisnychenko V, Plunkett G 3rd, Herring CD, Feher T, Posfai J, Blattner FR, Posfai G: Engineering a reduced Escherichia coli genome. Genome Res 2002, 12:640-647.

33. Hayashi K, Morooka N, Yamamoto Y, Fujita K, Isono K, Choi S, Ohtsubo E, Baba T, Wanner BL, Mori H, Horiuchi T: Highly accurate genome sequences of Escherichia coli K-12 strains MG1655 and W3110. Mol Syst Biol 2006, 2:0007.

34. Durfee T, Nelson R, Baldwin S, Plunkett G 3rd, Burland V, Mau B, Petrosino JF, Qin X, Muzny DM, Ayele M, et al: The complete genome sequence of Escherichia coli DH10B: insights into the biology of a laboratory workhorse. J Bacteriol 2008, 190:2597-2606.

doi:10.1186/1475-2859-11-110

Cite this article as: Billerbeck and Panke: A genetic replacement system for selection-based engineering of essential proteins. Microbial Cell Factories 2012 11:110

\section{Submit your next manuscript to BioMed Central and take full advantage of:}

- Convenient online submission

- Thorough peer review

- No space constraints or color figure charges

- Immediate publication on acceptance

- Inclusion in PubMed, CAS, Scopus and Google Scholar

- Research which is freely available for redistribution 